Peer

Physical Chemistry
Submitted 8 March 2021

Accepted 24 April 2021

Published 17 May 2021

Corresponding authors

Casper Steinmann, css@bio.aau.dk

Jan H. Jensen, jhjensen@chem.ku.dk

Academic editor

Johannes Margraf

Additional Information and Declarations can be found on page 11

DOI 10.7717/peerj-pchem.18

Copyright

2021 Steinmann and Jensen

Distributed under

Creative Commons CC-BY 4.0

OPEN ACCESS

\section{Using a genetic algorithm to find molecules with good docking scores}

\author{
Casper Steinmann ${ }^{1}$ and Jan H. Jensen ${ }^{2}$ \\ ${ }^{1}$ Department of Chemistry and Bioscience, Aalborg University, Aalborg, Denmark \\ ${ }^{2}$ Department of Chemistry, University of Copenhagen, Copenhagen, Denmark
}

\section{ABSTRACT}

A graph-based genetic algorithm (GA) is used to identify molecules (ligands) with high absolute docking scores as estimated by the Glide software package, starting from randomly chosen molecules from the ZINC database, for four different targets: Bacillus subtilis chorismate mutase (CM), human $\beta_{2}$-adrenergic $\mathrm{G}$ protein-coupled receptor $\left(\beta_{2} \mathrm{AR}\right)$, the DDR1 kinase domain (DDR1), and $\beta$-cyclodextrin (BCD). By the combined use of functional group filters and a score modifier based on a heuristic synthetic accessibility (SA) score our approach identifies between ca 500 and 6,000 structurally diverse molecules with scores better than known binders by screening a total of 400,000 molecules starting from 8,000 randomly selected molecules from the ZINC database. Screening 250,000 molecules from the ZINC database identifies significantly more molecules with better docking scores than known binders, with the exception of $\mathrm{CM}$, where the conventional screening approach only identifies 60 compounds compared to 511 with GA+Filter+SA. In the case of $\beta_{2} \mathrm{AR}$ and DDR1, the GA+Filter+SA approach finds significantly more molecules with docking scores lower than -9.0 and -10.0 . The GA+Filters+SA docking methodology is thus effective in generating a large and diverse set of synthetically accessible molecules with very good docking scores for a particular target. An early incarnation of the GA+Filter+SA approach was used to identify potential binders to the COVID-19 main protease and submitted to the early stages of the COVID Moonshot project, a crowd-sourced initiative to accelerate the development of a COVID antiviral.

Subjects Theoretical and Computational Chemistry

Keywords Genetic algorithm, Docking

\section{INTRODUCTION}

Docking of molecules to protein targets is an important part of computer aided drug discovery (Kitchen et al., 2004). One use of molecular docking is high throughput virtual screening (HTVS) of libraries of known molecules. Recent studies have show that such HTVS of hundreds of millions (Lyu et al., 2019) or even billions of molecules (Grebner et al. 2019) are possible. However, such large numbers pale in comparison with the estimated $10^{60}$ small molecules that make up chemical space.

The only practical way to search this space is to use search algorithms to identify interesting sub-spaces of manageable sizes. Historically, most work in this area as it relates to drug discovery have used evolutionary search algorithms to address this problem and such methods have also been applied to docking. The use of evolutionary algorithms 
in drug discovery has been reviewed by Devi, Sathya \& Coumar (2015). Examples involving docking include work by Pegg, Haresco \& Kuntz (2001), Nicolaou, Apostolakis \& Pattichis (2009), and Daeyaert \& Deem (2016) who all use genetic algorithms (GA) to optimise docking scores obtained by the DOCK (Ewing et al., 2001), Glamdock (Tietze \& Apostolakis, 2007), Autodoc-Vina (Trott \& Olson, 2010) programs, respectively. All three methods combine predefined molecular fragments in order to help ensure that the final molecules are synthetically accessible. Very recently, Cofala et al. (2020) and Nigam et al. (2020) presented SELFIES (Krenn et al., 2020)-based (mutations only) GA approaches for optimising docking scores. Cofala et al. (2020) optimised QuickVina 2 (Alhossary et al., 2015) docking scores for COVID-19 main protease ( $\left.\mathrm{M}^{\text {Pro }}\right)$. However, the several of the presented molecules in this study appear synthetically inaccessible. Nigam et al. (2020) optimised docking scores to 5-hydroxytryptamine receptor 1B and Cytochrome P450 2D6 by interpolating between a known binder to each target. Finally, Cieplinski et al. (2020) and Boitreaud et al. (2020) have used variational autoencoders (Kusner, Paige \& Hernández-Lobato, 2017; Gómez-Bombarelli et al., 2018) to optimise SMINA (Koes, Baumgartner \& Camacho, 2013) and Autodoc-Vina docking scores for several targets. Cieplinski et al. (2020) noted difficulties in finding good binders using this approach while Boitreaud et al. (2020) achieved some success.

In this paper we show that a non-fragment based GA (Jensen, 2019) can be used to find more synthetically accessible molecules with good Glide (Friesner et al., 2004; Halgren et al., 2004) docking scores compared to conventional HTVS of libraries. We note that our study does not address whether docking is useful for drug discovery.

\section{COMPUTATIONAL METHODOLOGY}

A graph-based genetic algorithm (Jensen, 2019) (GA) is used to identify molecules (ligands) with high absolute docking scores as estimated by the Glide software package (Friesner et al., 2004; Halgren et al., 2004) using either the faster HTVS or the slower SP scoring methodology. While the scoring functions for HTVS and SP are the same, SP samples more intermediate conformations throughout the docking funnel, and also reduces the thoroughness of the final torsional refinement and sampling. For the COVID Moonshot project we also rescore some ligands using the XP scoring function, which has greater requirements for ligand-receptor shape complementarity and weeds out false positives that SP lets through. Five conformations of each molecule are generated using RDKit and minimized with the MMFF94 force field (Halgren, 1996a; Halgren, 1996b; Halgren, 1996c; Halgren, 1996d; Halgren \& Nachbar, 1996). The lowest energy conformer is used for docking. The population size is 400 molecules, the mutation rate is $50 \%$ (meaning that a mutation operation is applied to $50 \%$ of the offspring-molecules), and the number of generations is 50 . The maximum molecule size allowed is $30 \pm 5$ non-hydrogen atoms. Molecules are chosen for mating with a probability proportional to their scores (roulette selection) and the 400 best-scoring molecules are advanced to the next generation (elitism). The initial population is chosen randomly from a 250,000-molecule subset of the ZINC database (Sterling \& Irwin, 2015) used in a previous study (Jensen, 2019). 
As noted by Gao \& Coley (2020) and Brown et al. (2019) generative models in general and GAs in particular often generate molecules with known chemically unstable bonds or molecules that are difficult to synthesise. We address this issue in three ways: we use Walters rd_filters code (following Brown et al. (2019)), a score modifier suggested by Gao \& Coley (2020) based on a heuristic synthetic accessibility (SA) score (Ertl \& Schuffenhauer, 2009), and a combination of the two. The rd_filers code contains several sets of SMARTS strings defining unstable bonds or groups. We use all the sets and eliminate any molecule with any of these moieties from the population. In the score modifier approach, the docking score is multiplied by a modified Gaussian function that ranges from 0 to 1 for high and low values of the SA score, respectively (a low SA score indicates a synthetically accessible molecule):

score $:=$ score $\cdot e^{-\frac{1}{2}\left(\frac{\max (\text { SA score }, \mu)-\mu}{\sigma}\right)^{2}}$

where $\mu=2.230044$ and $\sigma=0.6526308$ (Gao \& Coley, 2020). We found that the heuristic SA score depends on the protonation state of acid/base groups and is lower (better) for the neutral protonation state, so we neutralise such groups before computing the SA score.

The synthetic accessibility of the molecules in the final populations are estimated using the Molecule.one software package (Anonymous, 2020). The calculations are submitted remotely to Molecule.one servers using a license generously provided by Molecule.one for this project. Just as for the heuristic SA score it is important to supply this algorithm with the neutralised forms of the molecules.

The docking targets are Bacillus subtilis chorismate mutase (CM), human $\beta_{2}$-adrenergic G protein-coupled receptor ( $\left.\beta_{2} \mathrm{AR}\right)$, the DDR1 kinase domain (DDR1), and $\beta$-cyclodextrin (BCD). For the three proteins we use the 2CHT (Chook, Ke \& Lipscomb, 1993), 2RH1 (Cherezov et al., 2007), and 3ZOS (Canning et al., 2014) crystal structures from the Protein Data Bank (Berman, 2000), respectively. The proteins are prepared for docking with the Protein Preparation Wizard (Sastry et al., 2013) in the Maestro (Schrödinger, LLC, 2019) software by protonating all residues assuming pH 7 with PropKa (Olsson et al., 2011). All three protein structures contain co-crystallized ligands: a transition state analog (TSA) for $\mathrm{CM}$, carazolol for $\beta_{2} \mathrm{AR}$, and ponabtidin for DDR1. All water molecules in the crystal structures were removed before constructing a docking grid. For the three proteins, the position of each co-crystalized ligand is used as the centroid for the docking grid whereas for BCD we used the center of mass. A $20 \AA$ buffer around the centroid was employed when constructing the docking grid. We re-dock these ligands to their respectively targets to get an idea of what docking score one would expect for known binders. We determined the protonation state and overall charge of each ligand ( -2 for TSA, 0 for carazolol, and +1 for ponabtidin) by visual inspection of the crystal structure. In addition, we dock the known BCD-binder 6-(phenylamino)naphthalene-2-sulfonate (2,6-ANS) to BCD in the anionic form based on the typical $\mathrm{pKa}$ of the sulfonate group. The structures of the ligands are displayed in Table 1. 


\begin{tabular}{|c|c|c|c|c|c|c|}
\hline Target & Molecule & Charge & HTVS & SP & SA score & Molecule.one \\
\hline $\mathrm{CM}$ & TSA & -2 & -7.5 & -8.1 & 5.4 & 10.0 \\
\hline$\beta_{2} \mathrm{AR}$ & Carazolol & 0 & -6.8 & & 2.6 & 2.9 \\
\hline DDR1 & Ponatinib & +1 & -6.9 & & 2.9 & 2.3 \\
\hline $\mathrm{BCD}$ & 2,6-ANS & -1 & -4.4 & & 1.8 & 2.2 \\
\hline
\end{tabular}

Table 2 Columns 2-5 list the number of molecules (out of a total of about 8,000) with docking scores higher than known binders (Table 1 ) without any structural screening (GA), with the group-filters (+Filter), with a heuristic synthetic accessibility score (SA), and with both Filter and SA. The HTVS scoring methodology is used except for CM(SP) where the SP scoring methodology is used. Columns 6 and 7 list the corresponding number molecules with scores lower than $-9.0 \mathrm{kcal} / \mathrm{mol}$ and $-10.0 \mathrm{kcal} / \mathrm{mol}$ obtained with + Filter $+\mathrm{SA}$. The last three columns list the corresponding number of molecules obtained by docking all the molecules from the $250 \mathrm{~K}$ ZINC subset.

\begin{tabular}{|c|c|c|c|c|c|c|c|c|c|}
\hline & GA & +Filter & $+\mathrm{SA}$ & +Filter + SA & $<-9.0$ & $<-10.0$ & ZINC & $<-9.0$ & $<-10.0$ \\
\hline $\mathrm{CM}$ & 7,963 & 7,300 & 181 & 511 & 0 & 0 & 60 & 0 & 0 \\
\hline $\mathrm{CM}(\mathrm{SP})$ & 4,638 & & & & & & & & \\
\hline$\beta_{2} \mathrm{AR}$ & 7,994 & 7,879 & 2,493 & 2,125 & 164 & 10 & 16,262 & 86 & 1 \\
\hline DDR1 & 7,940 & 7,239 & 2,469 & 2,119 & 378 & 38 & 11,713 & 199 & 8 \\
\hline BCD & 8,000 & 7,947 & 6,214 & 6,218 & 0 & 0 & 152,209 & 0 & 0 \\
\hline
\end{tabular}

\section{RESULTS AND DISCUSSION}

We perform 20 different GA searches using the HTVS scoring function for each target. With a population size of 400 this generates up to 8,000 different potential binders for each target in the final populations (there are a few duplicates for some targets). Column 2 in Table 2 shows the number of molecules that have better (more negative) scores than known binders to the four targets (Table 1). Virtually all the molecules in the final populations have better scores than the known binders using the simpler HTVS scoring methodology. The average scores shown in Table 3 show that the scores are not only better, but considerably better, than for the known binders, except for CM where the decrease is more modest $(0.8 \mathrm{kcal} / \mathrm{mol}$ compared to $3.1-4.7 \mathrm{kcal} / \mathrm{mol})$. When using the more complex SP scoring function for CM the number of molecule with better scores drops to 4,638 compared to 7,963, indicating that any conclusions drawn below is likely to depend on the scoring function. However, given the computational expense of the SP scoring function and the relatively large number of docking simulations performed in this study we continue using the HTVS scoring function below.

While these results are encouraging, visual inspection of some of the best scoring molecules (first column of molecules in Fig. 1) show that they do not resemble drug-like 
Table 3 The average docking score and standard deviation (in $\mathrm{kcal} / \mathrm{mol}$ ) of all the molecules in the combined final populations of $20 \mathrm{GA}$ searches, except for "ZINC" which lists the corresponding values for the 8,000 top scoring molecules obtained using the $250 \mathrm{~K}$ ZINC subset.

\begin{tabular}{llllll} 
& GA & +Filter & +SA & +Filter + SA & ZINC \\
\hline CM & $-8.3 \pm 0.4$ & $-8.7 \pm 0.7$ & $-5.8 \pm 1.0$ & $-5.8 \pm 1.3$ & $-6.1 \pm 0.4$ \\
CM(SP) & $-8.2 \pm 0.3$ & & & & \\
$\beta_{2}$ AR & $-10.1 \pm 0.6$ & $-9.4 \pm 0.4$ & $-7.0 \pm 1.2$ & $-7.0 \pm 1.2$ & $-8.0 \pm 0.3$ \\
DDR 1 & $-10.0 \pm 0.6$ & $-9.9 \pm 0.5$ & $-6.6 \pm 1.5$ & $-6.4 \pm 1.6$ & $-7.7 \pm 0.5$ \\
BCD & $-9.1 \pm 0.2$ & $-8.2 \pm 0.5$ & $-5.6 \pm 0.8$ & $-5.3 \pm 0.8$ & $-5.7 \pm 0.2$ \\
\hline
\end{tabular}

molecules, indicating that they may be unstable and/or synthetically inaccessible. To quantify this property we compute a synthetic accessibility score using the Molecule.one retrosynthesis package which is based on machine learning algorithms and trained on a large number of reactions. Molecule.one returns a synthetic accessibility score ranging from 1 (very synthetically accessible) to 10 (not synthetically accessible) and the fraction of the 100 best-scoring molecules with a Molecule.one score below 10 is shown in Column 2 of Table 4 . DDR1 is the only case for which a non-negligible fraction of molecules $(26 \%)$ may be synthetically viable.

This problem has been observed before for generative models by, for example, Gao \& Coley (2020), Brown et al. (2019), and Renz et al. (2020). We address this issue in three ways: we use Walters rd_filters code (following Brown et al. (2019)), a score modifier based on a heuristic synthetic accessibility (SA) score (Ertl \& Schuffenhauer, 2009) suggested by Gao \& Coley (2020), and a combination of the two. The results are shown in Table 2 and show that the use of filters has relatively little effect on the number of molecules with better scores than the known binders and their average docking score. Unfortunately, there is also a negligible effect on the fraction of molecules deemed synthetically accessible molecules by Molecules.one (Table 4). Inspection of the best scoring molecules for each target (second column of molecules in Fig. 1) reveals that while the filters successfully prevented unstable bonding patterns, they do not prevent other reactive moieties such as cyclopentadienes and cyclohexadiene-like motifs.

The use of the SA score modifier has a much bigger effect on the number of molecules with better scores than the known binders and their average docking score. The effect is most pronounced for $\mathrm{CM}$ where the number of good binders drops more than an order of magnitude to only 181 molecules, while the decrease is about $70 \%$ for both $\beta_{2} \mathrm{AR}$ and DDR1 and 23\% for BCD. The most likely explanation is that ligands that bind well in the CM binding pocket tend to have high (bad) SA scores compared to the other targets. This is supported by the fact that the SA scores for the known binders TSA, carazolol, ponatinib, and 2,6-ANS (Table 1) are 5.4, 2.6, 2.9, and 1.8, respectively. The corresponding Molecule.one scores are 10.0, 2.9, 2.3, and 2.2, which indicates that the heuristic scores correlate well with the more sophisticated ML approach used by Molecule.one. With fewer molecules in the final population with high scores the average score necessarily increases (becomes less negative). The good news is that the fraction of molecules in the final population that Molecule.one deems synthetically accessible increases significantly to 


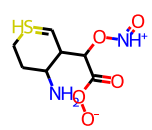

a. GA (-10.8)

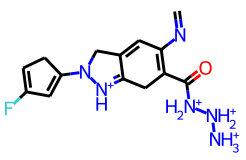

e. GA $(-13.3)$

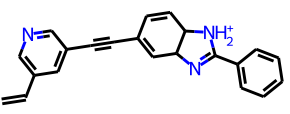

i. GA (-13.9)

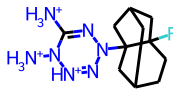

m. GA (-10.1)

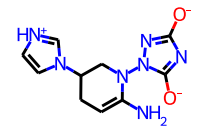

b. +Filter (-11.1)

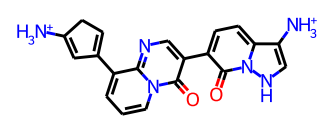

f. +Filter (-12.6)

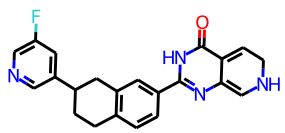

j. +Filter (-12.4)

k. +SA (-11.3)

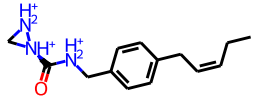

n. +Filter (-10.2)

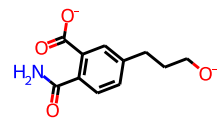

C. +SA (-8.9)

g. $+S A(-10.5)$

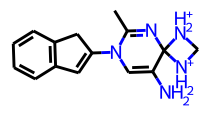

o. +SA (-8.5)

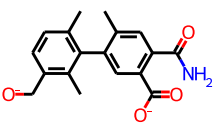

d. +Filter+SA (-8.9)
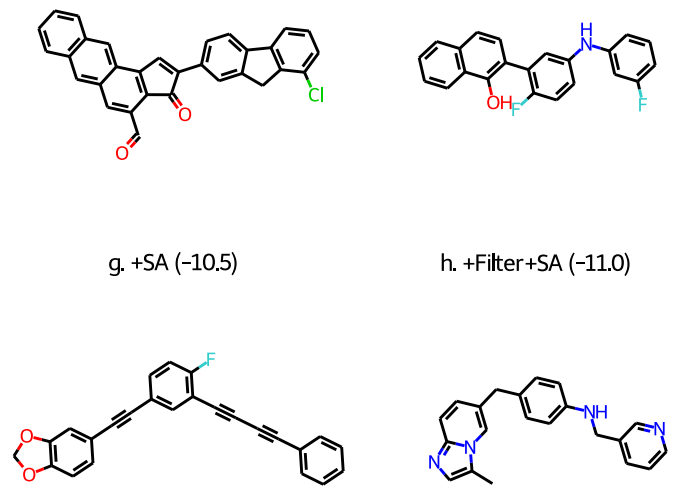

I. +Filter+SA (-11.6)

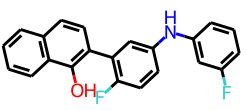

h. + Filter+SA (-11.0)

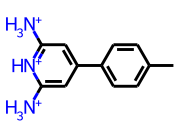

p. + Filter+SA (-7.8)

Figure 1 Best scoring molecules from the final population of GA-docking runs (see text for explanation) for the four different targets: CM (A-D), $\boldsymbol{\beta}_{2} \mathrm{AR}(\mathrm{E}-\mathrm{H})$, DDR1 (I-L), and BCD (M-P). The scores (in $\mathrm{kcal} / \mathrm{mol}$ ) are shown in parentheses.

Full-size DOI: 10.7717/peerjpchem.18/fig-1

between 0.24 to 0.64 . These fraction can be further increased to between 0.76 and 0.91 , with only negligible effect on the number of good binders in the final population, by using the score modifier together with the filters. The increase in good binders (to 511) in the case of CM is most likely due to the stochastic nature of the GA searches.

A plot of the Molecule.one score vs the docking score (Fig. 2A) obtained using Filters+SA shows no correlation. Better scoring molecules are thus not necessarily harder to synthesise and the top scoring molecules for each target all have relatively low (good) synthetic accessibility scores. Furthermore, the fractions of synthetically accessible molecules 
Table 4 Fraction of the 100 top scoring molecules that are deemed synthetically accessible by Molecule.one.

\begin{tabular}{llllll} 
& GA & +Filter & +SA & +Filter + SA & ZINC \\
\hline CM & 0.00 & 0.03 & 0.45 & 0.91 & 0.83 \\
$\beta_{2}$ AR & 0.00 & 0.05 & 0.55 & 0.76 & 0.84 \\
DDR1 & 0.26 & 0.29 & 0.64 & 0.88 & 0.82 \\
BCD & 0.03 & 0.02 & 0.24 & 0.85 & 0.89 \\
\hline
\end{tabular}
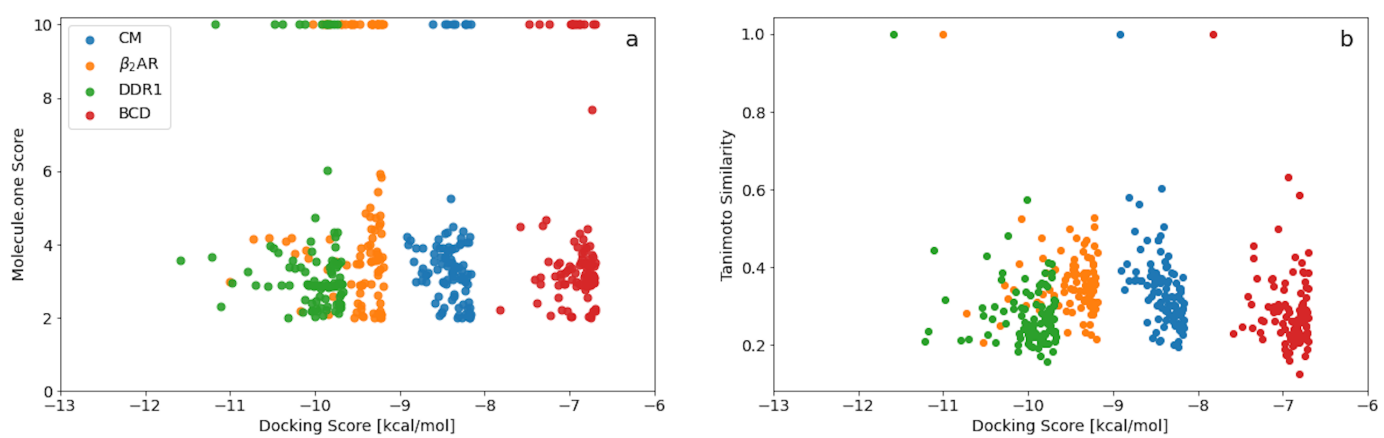

Figure 2 (A) Molecule.one score versus docking score for the 100 best binders predicted for the four different targets using Filters and SA. (B) ECFP4 Tanimoto similarity to the best scoring molecule for each target vs docking score for the $\mathbf{1 0 0}$ best binders predicted for the four different targets using Filters and SA.

Full-size DOI: 10.7717/peerjpchem.18/fig-2

computed using the top 100 scoring molecules are thus expected to be representative of the corresponding fractions for the entire final population.

A similar plot of the ECFP4 Tanimoto similarity to the best scoring molecule for each target vs docking score (Fig. 2B) also shows no correlation. The minimum and maximum similarity to the best scoring molecules are in the range of about $0.2-0.6$ and indicting a great deal of structural diversity among the 100 best scoring molecules for each target. The GA+Filters+SA docking methodology is thus effective in generating a large and diverse set of synthetically accessible molecules with high docking scores for a particular target.

Inspection of the top scoring molecules obtained with both filters and a score modifier (Fig. 1 (final column) and Fig. S2) reveal fairly ordinary looking organic molecules except that the charged states for CM and BCD (Figs. 1D and 1P) are not reasonable for a $\mathrm{pH}$ of 7. Future studies using this approach will need to correct this by, for example, including additional filters or adding a term to the score that penalizes large deviations from empirically estimated $\mathrm{pK}_{a}$ values.

Finally, while the accuracy of the chosen docking methodology is not a focus of this paper we do note some encouraging signs for the HTVS scoring function in Glide. For example, all the top scoring molecules for CM (Fig. 1) are dianions just like the known binder TSA (Fig. 1) and the CM substrate chorismate. Only 327 out of the 250,000 molecules in the ZINC subset that the initial population is randomly drawn from are dianions so the dianion motif is most likely generated by the GA. Similarly, for the fluorene moiety seen in both carazolol and Fig. $1 \mathrm{G}$ as well as two ethylene linked aromatic moieties seen in ponatinib 
and Figs. 1I and 1K, but with only 242 and 40 occurrences in the 250K ZINC subset, respectively. For BCD there is a clear preference for both lipophilic (adamantane-like in Fig. 1M) moieties favoring binding to the pocket as well as hydrophilic moieties which are also representative of the structures that bind favorably according to experiments.

\section{Comparison to conventional HTVS}

While the GA+Filter+SA results are encouraging they do involve the docking of 400,000 compounds and the question whether equally good results can be obtained by simply screening a library of molecules of similar size. To answer this question we dock all 250,000 molecules in the ZINC subset from which we sample the initial population for the GA searches. This approach identifies significantly more molecules with better docking scores than known binders, with the exception of CM, where the conventional HTVS approach only identifies 60 compounds compared to 511 with GA+Filter+SA (Table 2). However, the known binders for the remaining targets have relatively low docking scores of less than $-7.0 \mathrm{kcal} / \mathrm{mol}$. In the case of $\beta_{2} \mathrm{AR}$ and DDR1 the GA+Filter+SA approach finds significantly more molecules with docking scores lower than $-9.0 \mathrm{kcal} / \mathrm{mol}$ and -10.0 $\mathrm{kcal} / \mathrm{mol}$. Here, GA+Filter+SA finds 1.9 times as many molecules with a docking score lower than $-9.0 \mathrm{kcal} / \mathrm{mol}$ by docking only 1.6 times as many molecules. Overall, the number of molecules deemed synthetically accessible by Molecule.one is about the same as for GA+Filter+SA: somewhat higher for $\beta_{2} \mathrm{AR}$ and $\mathrm{BCD}$ and somewhat lower for $\mathrm{CM}$ and DDR1 (Table 4, the top-10 scoring molecules for each target is shown in Fig. S3). The GA+Filter+SA therefore seems like a promising approach for finding molecules with very good docking scores compared to conventional HTVS of libraries.

\section{The COVID Moonshot project}

The COVID Moonshot project (Chodera et al., 2020), a crowd-sourced initiative to accelerate the development of a COVID antiviral, was announced in mid-March 2020. The organizers of the project provided several crystal structures of the COVID-19 main protease $\left(\mathrm{M}^{\text {Pro }}\right)$ in complex with several small fragments (Douangamath et al., 2020) (Fig. S1) and invited the scientific community use this data to construct and submit potential $\mathrm{M}^{\text {Pro }}$ inhibitors for further experimental verification. Though we were in a relatively early stage of this project we decided to build upon our methodology as it was then, to construct candidates for the second and third rounds of submissions.

For Round 2 we perform 20 GA searches with a population size of 400 using the HTVS+Filter+SA as above and the 6LU7 crystal structure of $M^{\text {Pro }}$ (Jin et al., 2020). However, at that early stage we sampled our initial population from the first 1,000 molecules of the $250 \mathrm{~K}$ ZINC database subset. Also, we were not yet aware of the importance of neutralizing acid base groups before computing the SA score and checking synthetic accessibility, but this does not seem to be important for this target. Based on experience with the other targets we did increase the maximum molecule size to $50 \pm 5$ non-hydrogen atoms. All molecules in the final population are re-scored using the Glide XP scoring function (Friesner et al., 2006). The 128 molecules with a score better than or equal to $-7.0 \mathrm{kcal} / \mathrm{mol}$ are selected and subjected to retrosynthetic analysis using the ASKCOS 
software package (Coley et al., 2019) using the settings suggested by Gao \& Coley (2020). Molecules with less than 4 synthetic steps are selected and re-docked using the XP scoring methodology. The six molecules with XP scores better than $-7.5 \mathrm{kcal} / \mathrm{mol}$ (Figs. 3A)-3F were then submitted to Round 2 in March 30th, 2020. The feedback on Twitter was that the molecules were rather small and more fragment-like than drug-like.

For Round 3 we use Glide SP rather than HTVS for the GA search, use Molecule.one in addition to ASKCOS to determine synthetic accessibility for molecules with XP scores better than $-7.0 \mathrm{kcal} / \mathrm{mol}$, and eliminate all molecules with for which both ASKCOS and Molecule.one fail to find a retrosynthetic route. The remaining 136 molecules are then re-docked using the XP scoring methodology and molecules are selected from among the top-scoring molecules. We selected four molecules for submission to Round 3 (Figs. $3 G-3 J$ ) based on their score, diversity (also relative to our Round 2 submissions), and size and submitted them to Round 3 on April 2nd, 2020. (We also submitted four molecules selected based purely on their score, i.e., with synthetic accessibility considerations based on ASKCOS and Molecule.one, which are not discussed further).

Of the 10 submitted molecules, one was selected by the organizers (Fig. 3B) for synthesis and assay, but showed relatively low inhibition (10\% average inhibition at $20 \mu \mathrm{M})$ and was not pursued further. Many of our submissions feature a urea linkage or amide linkage that are also present in many of the fragment binders identified by the COVID Moonshot organizers (Fig. S1). In fact one of our submissions (Fig. S3F) differs by only a few atoms from one of the fragments (Fig. S1L) as well as one the submissions selected for further study. Overall, these results are quite encouraging given that our submissions are generated starting from randomly selected molecules.

\section{CONCLUSION AND OUTLOOK}

A graph-based genetic algorithm (Jensen, 2019) (GA) is used to identify molecules (ligands) with high absolute docking scores as estimated by the Glide software package (Friesner et al., 2004; Halgren et al., 2004), starting from randomly chosen molecules from the ZINC database. We perform 20 different GA searches using the HTVS scoring function each for four different targets: Bacillus subtilis chorismate mutase $(\mathrm{CM})$, human -adrenergic $\mathrm{G}$ protein-coupled receptor ( $\left.\beta_{2} \mathrm{AR}\right)$, the DDR1 kinase domain (DDR1), and $\beta$-cyclodextrin (BCD). With a population size of 400 this approach generates up to 8,000 different potential binders for each target, almost all of which have a better docking score than known binders (Figs. 1 and 2). However, many of these molecules do not resemble drug-like molecules (Fig. 1 and Fig. S2) and virtually none of the top-100 scoring molecules are deemed synthetically accessible by the retrosynthetic software package Molecule.one (Molecule.one, 2020) (Table 4).

Following suggestions by Brown et al. (2019) and Gao \& Coley (2020) we show that the synthetic accessibility can be increased significantly by the combined use of Walters rd_filters code and a score modifier based on a heuristic synthetic accessibility (SA) score (Ertl \& Schuffenhauer, 2009) (GA+Filter+SA). However, this also leads to a drop in the number of molecules with scores better than known binders of between 22\% (BCD) and 


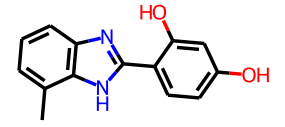

a. Round 2 (-8.0)<smiles>O=C(Nc1cc[nH]c1)Nc1cccc(F)c1</smiles>

c. Round 2 (-7.8)<smiles>Cc1ccc(NC(=O)Nc2cn[nH]c2)cc1C</smiles>

e. Round 2 (-7.6)<smiles>Clc1ccc(CNc2cccnc2)c(CNc2ccccn2)c1</smiles>

g. Round 3 (-7.7)<smiles>Cc1cccc(CCc2nc3cc(Cl)ccc3[nH]2)c1</smiles>

i. Round 3 (-7.3)<smiles>Cc1cc(C2Nc3ccccc3N2)ccc1O</smiles>

0.47<smiles>O=C(Nc1cccnc1)Nc1cccc(Cl)c1</smiles>

0.48<smiles>O=C(Cc1cccc(Cl)c1)Nc1cn[nH]c1</smiles>

0.42<smiles>O=C(Nc1cccnc1)c1cc(Cl)ccc1-c1ccccc1</smiles>

0.45

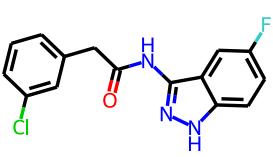

0.39<smiles>Cc1cc(-c2nc3ccccc3n2-c2ccc(O)c(C)c2)ccc1O</smiles><smiles></smiles>

b. Round 2 (-7.8)

1.0<smiles>O=C(Nc1ccc(Cl)cc1)Nc1cc[nH]c1</smiles>

d. Round 2 (-7.6)

0.46<smiles>Cc1ncccc1NC(=O)Nc1ccc(F)cc1</smiles><smiles>Cc1ncncc1NC(=O)Nc1ccccc1</smiles>

0.49<smiles>Cc1cc(NC2CCCC2)cc(C(=O)Nc2ccccc2)c1</smiles>

h. Round 3 (-7.6)

0.47<smiles>O=C(Nc1cn[nH]c1)Nc1cccc(-c2cccnc2)c1</smiles><smiles>O=C(Nc1cccnc1)Nc1cccc(Cl)c1</smiles>

0.56

Figure 3 Molecules submitted to the second and third round of the COVID moonshot project (with the corresponding XP docking score in parenthesis). Next to each submitted molecule is a molecule that was selected for further study by the project organizers that most closely matches our submissions (with the corresponding ECFP4 Tanimoto similarity below). All docking scores in kcal/mol.

Full-size DOI: 10.7717/peerjpchem.18/fig-3

95\% (CM). The GA+Filter+SA approach thus identifies between roughly 500 and 6000 structurally diverse (Table 2 and Fig. S2) molecules with scores better than known binders by screening a total of 400,000 molecules starting from 8000 randomly selected molecules from the ZINC database. However, screening 250,000 molecules from the ZINC database 
identifies significantly more molecules with better docking scores than known binders, with the exception of CM, where the conventional HTVS approach only identifies 60 compounds compared to 511 with GA+Filter+SA (Table 2). The known binders for the remaining targets have relatively low docking scores of less than $-7.0 \mathrm{kcal} / \mathrm{mol}$. In the case of $\beta_{2}$ AR and DDR1 the GA+Filter+SA approach finds significantly more molecules with docking scores lower than $-9.0 \mathrm{kcal} / \mathrm{mol}$ and $-10.0 \mathrm{kcal} / \mathrm{mol}$. The GA+Filters+SA docking methodology is thus effective in generating a large and diverse set of synthetically accessible molecules with very good docking scores for a particular target. However, for targets such as CM that predominantly binds charged ligands this approach will need to correct for unphysical protonation states by, for example, including additional filters or adding a term to the score that penalizes large deviations from empirically estimated $\mathrm{pK}_{a}$ values.

An early incarnation of the GA+Filter+SA approach was used to identify potential binders to the COVID-19 main protease $\left(\mathrm{M}^{\mathrm{Pro}}\right)$ and submitted to the early stages of the COVID Moonshot project (Chodera et al., 2020), a crowd-sourced initiative to accelerate the development of a COVID antiviral. Of the 10 submitted molecules, one was selected by the COVID Moonshot organizers (Fig. 3B) for synthesis and assay, but showed relatively low inhibition $(10 \%$ average inhibition at $20 \mu \mathrm{M})$ and was not pursued further. Many of our submissions feature a urea linkage or amide linkage that are also present in many of the fragment binders identified by the COVID Moonshot organizers (Fig. S1). In fact one of our submissions (Fig. 3F) differs by only a few atoms from one of the fragments (Fig. S1L) as well as one the submissions selected for further study. Overall, these results are quite encouraging given that our submissions are generated starting from randomly selected molecules.

As pointed out by Cieplinski et al. (2020) docking scores may also be used as a challenging test for generative models that "reflect the complexity of real discovery problems" (Coley, Eyke\& Jensen, 2020). Our study suggests that finding synthetically accessible molecules with good docking scores for CM presents an especially challenging objective function, and more so if the SP docking score is used. However, a benchmark based on a commercial software package such as Glide is not ideal and it remains to be seen whether this target is equally challenging using open source docking software such as SMINA (Koes, Baumgartner \& Camacho, 2013).

\section{ADDITIONAL INFORMATION AND DECLARATIONS}

\section{Funding}

The authors received no funding for this work.

\section{Competing Interests}

Jan H. Jensen is an Academic Editor for PeerJ. 


\section{Author Contributions}

- Casper Steinmann conceived and designed the experiments, performed the experiments, analyzed the data, performed the computation work, prepared figures and/or tables, authored or reviewed drafts of the paper, and approved the final draft.

- Jan H. Jensen conceived and designed the experiments, analyzed the data, performed the computation work, prepared figures and/or tables, authored or reviewed drafts of the paper, and approved the final draft.

\section{Data Availability}

The following information was supplied regarding data availability:

The code used in this study is available at GitHub: https://github.com/cstein/GB-

GA/tree/feature-glide_docking.

SMILES strings, docking scores, and Molecule.one scores are available at GitHub: https://github.com/cstein/GB-GA_docking_supporting_information.

\section{Supplemental Information}

Supplemental information for this article can be found online at http://dx.doi.org/10.7717/ peerj-pchem.18\#supplemental-information.

\section{REFERENCES}

Alhossary A, Handoko SD, Mu Y, Kwoh C-K. 2015. Fast, accurate, and reliable molecular docking with QuickVina 2. Bioinformatics 31(13):2214-2216 DOI 10.1093/bioinformatics/btv082.

Berman HM. 2000. The protein data bank. Nucleic Acids Research 28(1):235-242 DOI 10.1093/nar/28.1.235.

Boitreaud J, Mallet V, Oliver C, Waldispühl J. 2020. OptiMol: optimization of binding affinities in chemical space for drug discovery. Journal of Chemical Information and Modeling 60(12):5658-5666 DOI 10.1021/acs.jcim.0c00833.

Brown N, Fiscato M, Segler MH, Vaucher AC. 2019. GuacaMol: benchmarking models for de novo molecular design. Journal of Chemical Information and Modeling 59(3):1096-1108 DOI 10.1021/acs.jcim.8b00839.

Canning P, Tan L, Chu K, Lee SW, Gray NS, Bullock AN. 2014. Structural mechanisms determining inhibition of the collagen receptor DDR1 by selective and multitargeted type II kinase inhibitors. Journal of Molecular Biology 426(13):2457-2470 DOI 10.1016/j.jmb.2014.04.014.

Cherezov V, Rosenbaum DM, Hanson MA, Rasmussen S. GF, Thian FS, Kobilka TS, Choi H-J, Kuhn P, Weis WI, Kobilka BK, Stevens RC. 2007. High-resolution crystal structure of an engineered human 2-adrenergic G protein-coupled receptor. Science 318(5854):1258-1265 DOI 10.1126/science.1150577.

Chodera J, Lee AA, London N, Von Delft F. 2020. Crowdsourcing drug discovery for pandemics. Nature Chemistry 12(7):581-581 DOI 10.1038/s41557-020-0496-2.

Chook YM, Ke H, Lipscomb WN. 1993. Crystal structures of the monofunctional chorismate mutase from Bacillus subtilis and its complex with a transition state 
analog. Proceedings of the National Academy of Sciences of the United States of America 90(18):8600-8603 DOI 10.1073/pnas.90.18.8600.

Cieplinski T, Danel T, Podlewska S, Jastrzebski S. 2020. We should at least be able to design molecules that dock well. ArXiv preprint. arXiv:2006.16955.

Cofala T, Elend L, Mirbach P, Prellberg J, Teusch T, Kramer O. 2020. Evolutionary multi-objective design of SARS-CoV-2 protease inhibitor candidates. In: Parallel problem solving from nature-PPSN XVI. Zurich: Springer International Publishing, 357-371.

Coley CW, Eyke NS, Jensen KF. 2020. Autonomous discovery in the chemical sciences art II: outlook. Angewandte Chemie International Edition 59(52):23414-23436 DOI 10.1002/anie.201909989.

Coley CW, Thomas DA, Lummiss J. AM, Jaworski JN, Breen CP, Schultz V, Hart T, Fishman JS, Rogers L, Gao H, Hicklin RW, Plehiers PP, Byington J, Piotti JS, Green WH, Hart AJ, Jamison TF, Jensen KF. 2019. A robotic platform for flow synthesis of organic compounds informed by AI planning. Science 365(6453):eaax1566 DOI 10.1126/science.aax1566.

Daeyaert F, Deem MW. 2016. A pareto algorithm for efficient de novo design of multifunctional molecules. Molecular Informatics 36(1-2):1600044.

Devi RV, Sathya SS, Coumar MS. 2015. Evolutionary algorithms for de novo drug design -a survey. Applied Soft Computing 27:543-552

DOI 10.1016/j.asoc.2014.09.042.

Douangamath A, Fearon D, Gehrtz P, Krojer T, Lukacik P, Owen CD, Resnick E, Strain-Damerell C, Aimon A, Ábrányi-Balogh P, Brandão-Neto J, Carbery A, Davison G, Dias A, Downes TD, Dunnett L, Fairhead M, Firth JD, Jones SP, Keeley A, Keserü GM, Klein HF, Martin MP, Noble MEM, O’Brien P, Powell A, Reddi RN, Skyner R, Snee M, Waring MJ, Wild C, London N, Von Delft F, Walsh MA. 2020. Crystallographic and electrophilic fragment screening of the SARS-CoV-2 main protease. Nature Communications 11(1):5047.

Ertl P, Schuffenhauer A. 2009. Estimation of synthetic accessibility score of drug-like molecules based on molecular complexity and fragment contributions. Journal of Cheminformatics 1(1):8.

Ewing TJ, Makino S, Skillman AG, Kuntz ID. 2001. DOCK 4.0: Search strategies for automated molecular docking of flexible molecule databases. Journal of ComputerAided Molecular Design 15(5):411-428 DOI 10.1023/A:1011115820450.

Friesner RA, Banks JL, Murphy RB, Halgren TA, Klicic JJ, Mainz DT, Repasky MP, Knoll EH, Shelley M, Perry JK, Shaw DE, Francis P, Shenkin PS. 2004. Glide: A new approach for rapid, accurate docking and scoring. 1. Method and assessment of docking accuracy. Journal of Medicinal Chemistry 47(7):1739-1749 DOI 10.1021/jm0306430.

Friesner RA, Murphy RB, Repasky MP, Frye LL, Greenwood JR, Halgren TA, Sanschagrin PC, Mainz DT. 2006. Extra precision glide: Docking and scoring incorporating a model of hydrophobic enclosure for protein-ligand complexes. Journal of Medicinal Chemistry 49(21):6177-6196 DOI 10.1021/jm051256o. 
Gao W, Coley CW. 2020. The synthesizability of molecules proposed by generative models. Journal of Chemical Information and Modeling 60:5714-5723 DOI 10.1021/acs.jcim.0c00174.

Gómez-Bombarelli R, Wei JN, Duvenaud D, Hernández-Lobato JM, Sánchez-Lengeling B, Sheberla D, Aguilera-Iparraguirre J, Hirzel TD, Adams RP, Aspuru-Guzik A. 2018. Automatic chemical design using a data-driven continuous representation of molecules. ACS Central Science 4(2):268-276 DOI 10.1021/acscentsci.7b00572.

Grebner C, Malmerberg E, Shewmaker A, Batista J, Nicholls A, Sadowski J. 2019. Virtual screening in the cloud: how big is big enough? Journal of Chemical Information and Modeling 60(9):4274-4282.

Halgren TA. 1996a. Merck molecular force field. I. Basis, form, scope, parameterization, and performance of MMFF94. Journal of Computational Chemistry 17(5-6):490-519 DOI 10.1002/(SICI)1096-987X(199604)17:5/6<490::AID-JCC1>3.0.CO;2-P.

Halgren TA. 1996b. Merck molecular force field. II. MMFF94 van der Waals and electrostatic parameters for intermolecular interactions. Journal of Computational Chemistry 17(5-6):520-552 DOI 10.1002/(SICI)1096-987X(199604)17:5/6<520::AID-JCC2>3.0.CO;2-V

Halgren TA. 1996c. Merck molecular force field. III. Molecular geometries and vibrational frequencies for MMFF94. Journal of Computational Chemistry 17(56):553-586

DOI 10.1002/(SICI)1096-987X(199604)17:5/6<553::AID-JCC3>3.0.CO;2-T.

Halgren TA. 1996d. Merck molecular force field. V. Extension of MMFF94 using experimental data, additional computational data, and empirical rules. Journal of Computational Chemistry 17(5-6):616-641

DOI 10.1002/(SICI)1096-987X(199604)17:5/6<616::AID-JCC5>3.0.CO;2-X.

Halgren TA, Murphy RB, Friesner RA, Beard HS, Frye LL, Pollard WT, Banks JL. 2004. Glide: a new approach for rapid, accurate docking and scoring. 2. Enrichment factors in database screening. Journal of Medicinal Chemistry 47(7):1750-1759 DOI 10.1021/jm030644s.

Halgren TA, Nachbar RB. 1996. Merck molecular force field. IV. conformational energies and geometries for MMFF94. Journal of Computational Chemistry 17(56):587-615.

Jensen JH. 2019. A graph-based genetic algorithm and generative model/Monte Carlo tree search for the exploration of chemical space. Chemical Science 10(12):3567-3572 DOI 10.1039/C8SC05372C.

Jin Z, Du X, Xu Y, Deng Y, Liu M, Zhao Y, Zhang B, Li X, Zhang L, Peng C, Duan Y, Yu J, Wang L, Yang K, Liu F, Jiang R, Yang X, You T, Liu X, Yang X, Bai F, Liu H, Liu X, Guddat LW, Xu W, Xiao G, Qin C, Shi Z, Jiang H, Rao Z, Yang H. 2020. Structure of Mpro from SARS-CoV-2 and discovery of its inhibitors. Nature 582(7811):289-293 DOI 10.1038/s41586-020-2223-y.

Kitchen DB, Decornez H, Furr JR, Bajorath J. 2004. Docking and scoring in virtual screening for drug discovery: methods and applications. Nature Reviews Drug Discovery 3(11):935-949 DOI 10.1038/nrd1549. 
Koes DR, Baumgartner MP, Camacho CJ. 2013. Lessons learned in empirical scoring with smina from the CSAR 2011 benchmarking exercise. Journal of Chemical Information and Modeling 53(8):1893-1904 DOI 10.1021/ci300604z.

Krenn M, Häse F, Nigam A, Friederich P, Aspuru-Guzik A. 2020. Self-referencing embedded strings (SELFIES): A 100\% robust molecular string representation. Machine Learning: Science and Technology 1(4):045024.

Kusner MJ, Paige B, Hernández-Lobato JM. 2017. Grammar variational autoencoder. ArXiv preprint. arXiv:1703.01925.

Lyu J, Wang S, Balius TE, Singh I, Levit A, Moroz YS, O'Meara MJ, Che T, Algaa E, Tolmachova K, Tolmachev AA, Shoichet BK, Roth BL, Irwin JJ. 2019. Ultralarge library docking for discovering new chemotypes. Nature 566(7743):224-229 DOI 10.1038/s41586-019-0917-9.

Molecule.one. 2020. Molecule.one retrosynthesis planning software. Available at https: //molecule.one/ (accessed on 22 December 2020).

Nicolaou CA, Apostolakis J, Pattichis CS. 2009. De novo drug design using multiobjective evolutionary graphs. Journal of Chemical Information and Modeling 49(2):295-307 DOI 10.1021/ci800308h.

Nigam A, Pollice R, Krenn M, Dos Passos Gomes G, Aspuru-Guzik A. 2020. Beyond generative models: superfast traversal, optimization, novelty, exploration and discovery (STONED) algorithm for molecules using SELFIES. Chemical Science 2021(Advance Article): DOI 10.1039/D1SC00231G.

Olsson MHM, Søndergaard CR, Rostkowski M, Jensen JH. 2011. PROPKA3: consistent treatment of internal and surface residues in empirical pKa predictions. Journal of Chemical Theory and Computation 7(2):525-537 DOI 10.1021/ct100578z.

Pegg SC-H, Haresco JJ, Kuntz ID. 2001. A genetic algorithm for structure-based de novo design. Journal of Computer-Aided Molecular Design 15(10):911-933 DOI 10.1023/A:1014389729000.

Renz P, Rompaey DV, Wegner JK, Hochreiter S, Klambauer G. 2020. On failure modes of molecule generators and optimizers. ChemRxiv DOI 10.26434/chemrxiv.12213542.

Sastry GM, Adzhigirey M, Day T, Annabhimoju R, Sherman W. 2013. Protein and ligand preparation: parameters, protocols, and influence on virtual screening enrichments. Journal of Computer-Aided Molecular Design 27(3):221-234 DOI 10.1007/s10822-013-9644-8.

Schrödinger, LLC. 2019. Schrödinger Release 2019-4, Maestro. New York: Schrödinger, LLC.

Sterling T, Irwin JJ. 2015. ZINC 15 -ligand discovery for everyone. Journal of Chemical Information and Modeling 55(11):2324-2337 DOI 10.1021/acs.jcim.5b00559.

Tietze S, Apostolakis J. 2007. GlamDock: development and validation of a new docking tool on several thousand protein-ligand complexes. Journal of Chemical Information and Modeling 47(4):1657-1672 DOI 10.1021/ci7001236.

Trott O, Olson AJ. 2010. AutoDock Vina: improving the speed and accuracy of docking with a new scoring function, efficient optimization, and multithreading. Journal of Computational Chemistry 31(2):455-461. 